\title{
Zirconium-containing UiO-66 as an efficient and reusable catalyst for transesterification of triglyceride with methanol
}

\author{
Fan Zhou ${ }^{\mathrm{a}}$, Ningyue Lu ${ }^{\mathrm{a}}$, Binbin Fan ${ }^{\mathrm{a}, *}$, Huigang Wang ${ }^{\mathrm{a}}$, Ruifeng $\mathrm{Li}^{\mathrm{b}, *}$ \\ ${ }^{a}$ College of Chemistry and Chemical Engineering, Taiyuan University of Technology, Taiyuan 030024, \\ Shanxi, China \\ ${ }^{\mathrm{b}}$ Key Laboratory of Coal Science and Technology MOE, Taiyuan University of Technology, Taiyuan \\ 030024, Shanxi, China \\ Article history: \\ Received 26 February 2016
}

Revised 29 April 2016

Accepted 20 June 2016

Available online

\begin{abstract}
Zirconium-based MOFs of the UiO family have attracted considerable attention due to their high thermal, chemical and mechanical stability. With the aim of further exploring the applications of zirconium-based UiO-66 in acid-catalyzed reactions and elucidating the effects of the defects in UiO-66 materials on their catalytic performances, in this work, a series of zirconium-containing UiO-66 samples were synthesized by varying the synthesis temperatures and $\mathrm{BDC} / \mathrm{Zr}$ (terephthalic acid/ $\mathrm{ZrCl}_{4}$ ) ratios in the synthesis system. The synthesized UiO-66 samples were characterized by X-ray diffraction (XRD), $\mathrm{N}_{2}$ adsorption-desorption, scanning electron microscopy (SEM), thermogravimetrical analysis (TGA), temperature-programmed desorption of $\mathrm{NH}_{3}\left(\mathrm{NH}_{3}\right.$-TPD). Their catalytic performances were investigated in transesterification of tributyrin and soybean oil with methanol. The results showed that UiO-66 samples with different amounts of defects could be successfully prepared by varying the synthesis temperatures and/or the $\mathrm{BDC} / \mathrm{Zr}$ ratios used in the synthesis system. The catalytic activities of the UiO-66 materials greatly depended on their linker defects and enhanced with the increase of the defect amount. The UiO-66 was an efficient catalyst for transesterification of tributyrin and soybean oil with methanol under mild reaction conditions and its catalytic activity was comparable to other solid acid catalysts reported in the literatures. The UiO-66 catalyst was relatively stable and could be reused.
\end{abstract}


Keywords: Metal organic frameworks; Solid acid; Transesterification; UiO-66; Catalyst

*Corresponding authors. Tel: +86-351-6010384; Fax: +86-351-6010121; E-mail: fanbinbin@tyut.edu.cn(B.B.Fan); rfli@tyut.edu.cn (R.F. Li).

This work is supported by the National Science Foundation of China (No. 20971095, 21576177), and Research Project Supported by Shanxi Scholarship Council of China (2013-047).

\section{Introduction}

Biodiesel is defined as alkyl esters of fatty acids derived from renewable biological products such as vegetable oils and animal fats. Biodiesel contains no sulfur and aromatic species, contributes much less to global warming than fossil fuels, and thereby is an attractive alternative to diesel usually produced from petroleum. Biodiesel can be produced by transesterification of triglycerides to methyl esters with homogeneous or heterogeneous strong base or acid as catalysts [1-3]. Compared with acid catalysts, alkaline catalysts are more effective and less corrosive, thus, commercially, homogeneous alkaline catalysts, such as $\mathrm{NaOH}$ or $\mathrm{KOH}$ are usually employed [4]. Nevertheless, homogenous alkaline catalysts suffer from many disadvantages, including catalyst separation, alkaline wastewater neutralization, and the salt (coming from neutralization step) contamination on glycerol by-product [5]. In addition, alkaline catalyst is sensitive to acid and cannot be used to treat low quality feedstock with high FFA content $(>0.5$ $w t \%$ of free fatty acids (FFA)) [6,7]. Therefore, the use of solid acid catalysts instead of homogeneous alkaline catalysts is preferred $[8,9]$. Sulfated or tungstated zirconia and supported heteropolyacids are the most used solid acids for biodiesel production by transesterification [10-12]. However, these solid acid catalysts often face the following problems: (1) large mass transfer resistance due to lack of large textural properties; (2) facile deactivation under the reaction conditions; (3) the difficulty in catalyst reuse. Sulfonic functionalized ion-exchange resins are also attractive solid acid catalysts but their low thermal stabilities limit their use at high reaction temperatures [13]. Hence, the development of an efficient and reusable heterogeneous solid acid catalyst for biodiesel production is highly desirable.

Metal organic frameworks (MOFs) are a class of interesting three-dimensional crystalline porous materials constructed by copolymerization of multidentate organic ligands with transition metal ions or metal ion clusters. MOFs, due to their inherent large surface areas, uniform but tunable cavities and tailorable chemistry, have shown wide potential applications in gas storage and separation, molecular recognition and catalysis [14-16]. Among a large number of MOFs, zirconium-based MOFs of the UiO 
family have attracted considerable attention due to their high thermal, chemical and mechanical stability [17-20]. UiO-66 with inorganic $\left[\mathrm{Zr}_{6} \mathrm{O}_{4}(\mathrm{OH})_{4}\right]^{12+}$ brick and terephthalate linkers is considered to be the parent of the other UiO members, from which other members are derived via isoreticular synthesis [17]. Zr-containing UiO-66 type MOFs usually display well Lewis acid character and have exhibited excellent catalytic performances in various acid-catalyzed reactions [21-25]. For example, Vermoortele and his co-workers have used UiO-66 as catalyst for citronellal cyclization [22]. Cirujano and his co-workers have demonstrated that UiO-66 and UiO-66- $\mathrm{NH}_{2}$ are active and stable catalysts for the acid-catalyzed esterification of various saturated fatty acids with $\mathrm{MeOH}$ and $\mathrm{EtOH}$ with activities comparable (in some cases superior) to other solid acid catalysts previously reported in literature [24]. These exciting results inspire us to further explore the application of zirconium-based MOFs in other acid-catalyzed reactions.

In this work, we synthesized a series of UiO-66 samples with different amounts of linker defects by varying the synthesis temperatures and $\mathrm{BDC} / \mathrm{Zr}$ (terephthalic acid/ $/ \mathrm{rCl}_{4}$ ) ratios in the synthesis system and investigated their catalytic performances in transesterification of tributyrin and soybean oil with methanol. The results show that UiO-66 is an efficient and reusable catalyst for transesterification reaction and the ligand defects have a great influence on its catalytic performance.

\section{Experimental}

\subsection{Synthesis of UiO-66 samples}

The UiO-66 samples were synthesized according to the method reported in the literature [26]. Typically, $\mathrm{ZrCl}_{4}(1.17 \mathrm{~g}, 5 \mathrm{mmol}), \mathrm{HCl}(0.8 \mathrm{~mL}, 37 \%)$ and terephthalic acid (BDC, $\left.0.83 \mathrm{~g}, 5 \mathrm{mmol}\right)$ were added to $30 \mathrm{~mL}$ of DMF (N,N'-dimethyl formamide). The resulting mixture was transferred to a $100 \mathrm{~mL}$ Teflon-liner stainless steel autoclave. The autoclave was sealed and heated at $120{ }^{\circ} \mathrm{C}$ for $20 \mathrm{~h}$. The material recovered by filtration was firstly washed thoroughly with fresh DMF for three times, and then extracted with methanol to completely remove residue DMF. Finally, the obtained solid was dried under vacuum at $200{ }^{\circ} \mathrm{C}$ for $3 \mathrm{~h}$. The other samples were also prepared according to the similar procedure except for the different synthesis temperatures or the BDC/Zr ratios. Table 1 summarized the sample names and the synthetic conditions. The synthesized samples were denoted as UiO-66- $x-y$, in which $x$ was the synthesis temperature and $y$ was the $\mathrm{BDC} / \mathrm{Zr}$ ratios in the synthesis system.

\subsection{Characterization of sample}


Powder X-ray diffraction (XRD) patterns were obtained on a Rigaku D/max-2500 diffractometer with $\mathrm{Cu} K \alpha$ radiation. Thermogravimetric measurement was performed on a Netzch SAT449 F3 thermal analysis system with an elevating rate of $10{ }^{\circ} \mathrm{C} / \mathrm{min}$ from $50{ }^{\circ} \mathrm{C}$ to $700{ }^{\circ} \mathrm{C}$ in air atmosphere. $\mathrm{N}_{2}$ physisorption isotherms were performed at $-196{ }^{\circ} \mathrm{C}$ using a Quantachrone Nova 1200e. Prior to measurement, the sample was evacuated at $300{ }^{\circ} \mathrm{C}$ for $3 \mathrm{~h}$. The surface area was calculated by BET method at relative pressure of $P / P_{0}=0.05-0.25$. Scanning electron microscopy (SEM) was performed using a Hitachi 4800. $\mathrm{NH}_{3}$-TPD measurement was performed using AutoChem 2720 instrument with $\mathrm{He}$ as the carries gas $(30 \mathrm{~mL} / \mathrm{min})$. First, $0.1 \mathrm{~g}$ of catalyst was charged into a quartz tube, and then pretreated at $300{ }^{\circ} \mathrm{C}$ for $3 \mathrm{~h}$. After being cooled to $50{ }^{\circ} \mathrm{C}$, the sample was saturated with dried $\mathrm{NH}_{3}$, and then blown with $\mathrm{He}$ to remove the physical-adsorbed $\mathrm{NH}_{3}$. Finally, desorption process was started from $50{ }^{\circ} \mathrm{C}$ to 350 ${ }^{\circ} \mathrm{C}$ at a heating rate of $10{ }^{\circ} \mathrm{C} / \mathrm{min}$ and monitored by a thermal conductivity detector.

Table 1. The names of the synthesized UiO-66 samples and the synthesis conditions.

\begin{tabular}{cccc}
\hline Sample & $\begin{array}{c}\mathrm{ZrCl}_{4} \\
(\mathrm{mmol})\end{array}$ & $\begin{array}{r}\text { Terephthalic acid } \\
(\mathrm{mmol})\end{array}$ & $\begin{array}{c}\text { Synthesis temperature } \\
\left({ }^{\circ} \mathrm{C}\right)\end{array}$ \\
\hline UiO-66-100-1 & 5 & 5 & 100 \\
UiO-66-100-2 & 5 & 10 & 100 \\
UiO-66-160-2 & 5 & 10 & 160 \\
UiO-66-220-2 & 5 & 10 & 220 \\
\hline
\end{tabular}

\subsection{Catalytic reaction}

The transesterification of tributyrin was carried out in a Teflon-lined autoclave in an oil bath. For transesterification of tributyrin, $1.3 \mathrm{mmol}$ of tributyrin (Aldrich, 98\%) was dissolved in a specified quantity of methanol, and then the mixture was put into the reactor containing $0.1 \mathrm{~g}$ of catalyst. The reaction mixture was magnetically stirred at $800 \mathrm{rpm}$ at the designed temperature. The product analysis was conducted on a SHIMADZU GC-2014 gas chromatograph equipped with a FID detector and an Rtx-1 capillary column with chlorobenzene as the internal standard.

The transesterification of soybean oil were also conducted in a Teflon-lined autoclave. $1.41 \mathrm{~g}$ (44 mmol) of methanol, $0.9 \mathrm{~g}$ (about $1.1 \mathrm{mmol}$ ) of soybean oil and $0.1 \mathrm{~g}$ of catalyst were added into the reactor, and then the reaction mixture was magnetically stirred at $800 \mathrm{rpm}$ at $140{ }^{\circ} \mathrm{C}$ for $5 \mathrm{~h}$. After finishing the reaction, the solid catalyst was quickly removed from the reaction mixture by filtration. The 
un-reacted methanol in the reaction mixture was removed via vacuum distillation. The glycerol and methyl fatty esters were analyzed by the gas chromatograph equipped with a FID detector and an Agilent-DB-5ht capillary separation column.

\section{Results and discussion}

\subsection{Sample characterization}

For UiO-66 material, its catalytic activity is mainly ascribed to missing linkers in the structure $[27,28]$. As missing linkers create open $\mathrm{Zr}$ sites which are accessible for catalytic transformations, different procedures, including utilizing modulator molecules (such as acetic acid and formic acid) and varying synthesis conditions, are developed to increase these structural defects within the UiO-66 type materials $[28,29]$. Recently, Shearer and his co-workers systemly studied the effect of synthesis temperatures and linker concentrations on the stability and defects of UiO-66 and found that increasing the synthesis temperatures and/or the $\mathrm{BDC} / \mathrm{Zr}$ ratios aided the "ironing out" of linker defects [29]. Based on their results, in this work, we synthesized a series of UiO-66 samples at different synthesis temperatures and $\mathrm{BDC} / \mathrm{Zr}$ ratios with the aim of elucidating the defects in UiO-66 materials on their catalytic performances in transesterification. The XRD patterns (Figure 1) of all the samples were in good agreement with the previous reports [17], confirming the formation of pure crystalline UiO-66 phase. However, their relative crystallinities were different and the samples prepared at higher synthesis temperatures or $\mathrm{BDC} / \mathrm{Zr}$ ratios had higher crystallinities. This result is in agreement with that observed by Shearer and his co-workers [29] and is linked to the defects resulted from linker missing. Shearer and his co-workers establish a method to evaluate the linker missing degree based on the TG results. They consider that the composition of perfect dehydroxylated UiO-66 is $\mathrm{Zr}_{6} \mathrm{O}_{6}\left(\mathrm{C}_{8} \mathrm{H}_{4} \mathrm{O}_{4}\right)_{6}$ and the molecular weight of $\mathrm{Zr}_{6} \mathrm{O}_{6}\left(\mathrm{C}_{8} \mathrm{H}_{4} \mathrm{O}_{4}\right)_{6}$ should be a factor of 2.2 higher than $6 \mathrm{ZrO}_{2}$ (the only solid product) in theory. Therefore, the defect formulation can be calculated by analyzing the mass loss in the decomposition step progressing from a formula of $\mathrm{Zr}_{6} \mathrm{O}_{6+x}\left(\mathrm{C}_{8} \mathrm{H}_{4} \mathrm{O}_{4}\right)_{6-x}$ at $300{ }^{\circ} \mathrm{C}$ to $6 \mathrm{ZrO}_{2}$ at $550{ }^{\circ} \mathrm{C}$ with an expected UiO-66/6 $\mathrm{ZrO}_{2}$ ratio of 2.2 for $x=0$. Based on their method and the mass loss obtained from Figure 2, the $x$ values for UiO-66-100-1, UiO-66-100-2, UiO-66-160-2 and UiO-66-220-2 four samples were 2.2, 1.7, 0.6 and 0.2 , respectively. The results showed that UiO-66 samples with different amounts of defects were successfully prepared by varying the synthesis temperatures and/or the $\mathrm{BDC} / \mathrm{Zr}$ ratios used in the synthesis system. The $\mathrm{N}_{2}$ adsorption-desorption isotherms of the different UiO-66 samples is displayed 
in Figure 3. It could be seen that nitrogen adsorption capacity varied depending on the synthesis parameters, and the UiO-66-100-1 sample with the most linker defects showed the highest BET surface area. The SEM images of the different UiO-66 samples (Figure 4) showed that all samples were crystallized well and have similar crystal sizes, even if there were some differences on their morphologies.

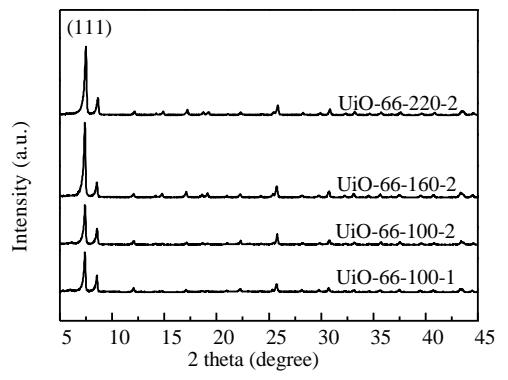

Figure 1. XRD patterns of the different UiO-66 samples.

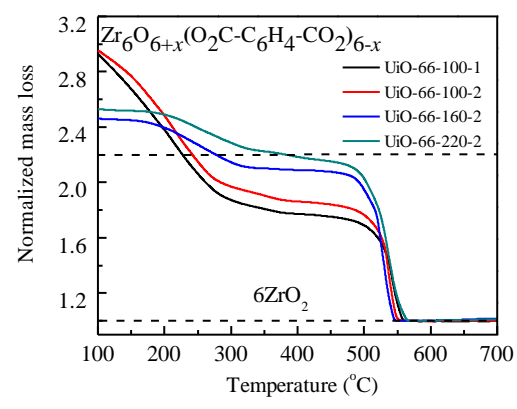

Figure 2. Normalized TGA thermal curves for samples collected under air flow, normalized to the mass of the $\mathrm{ZrO}_{2}$ decomposition product. A normalized mass loss of 2.2 corresponds to a defect free sample ( $x$ $=0)$.

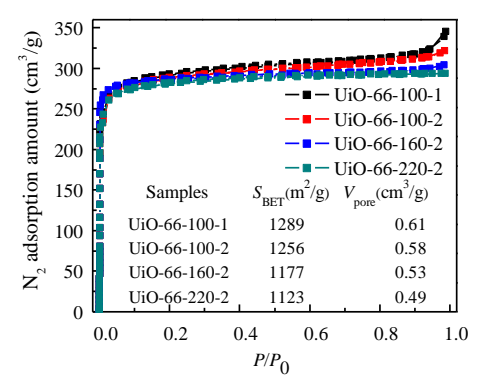

Figure 3. $\mathrm{N}_{2}$ isotherms of the different UiO-66 samples. 


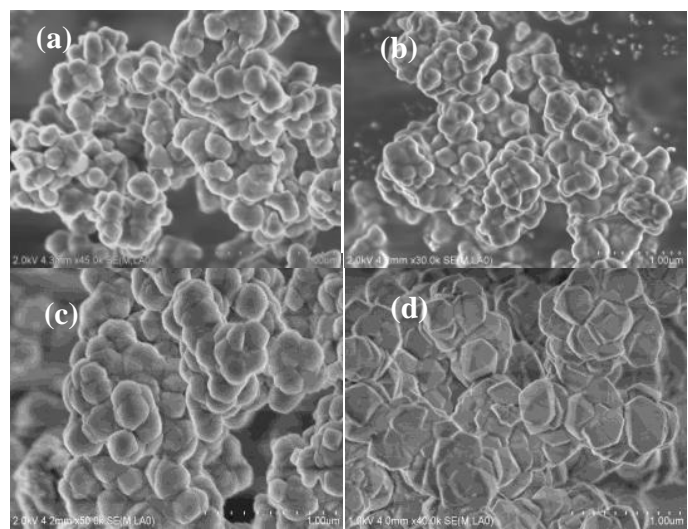

Figure 4. The SEM images of different samples. (a) UiO-66-100-1, (b) UiO-66-100-2, (c) UiO-66-160-2, (d) UiO-66-220-2.

$\mathrm{NH}_{3}$-TPD profiles over the different UiO-66 samples are illustrated in Figure 5. It can be seen that the $\mathrm{BDC} / \mathrm{Zr}$ ratios and synthesis temperatures have great influences on the acid characters of the synthesized UiO-66 samples. For UiO-66 material, its inorganic $\left[\mathrm{Zr}_{6} \mathrm{O}_{4}(\mathrm{OH})_{4}\right]^{12+}$ cluster will dehydroxylated to $\mathrm{Zr}_{6} \mathrm{O}_{6}$ inner cluster upon heating at $250{ }^{\circ} \mathrm{C}-300{ }^{\circ} \mathrm{C}$ [30], thus the acid properties of the different UiO-66 samples measured by $\mathrm{NH}_{3}$-TPD mainly involve their Lewis acid characters. The Lewis acid properties of the different UiO-66 samples are closely related to the amount of defects in their framework and the linker missing fashion [27]. UiO-66-220-2 sample only showed weak acidity, whereas other three samples showed weak to medium acidity. Moreover, compared the specific peak areas of different TPD profiles listed in Table 2, it could be seen that both the total acid amounts and the acid amount with the medium strength in the samples decreased with the increase of BDC/Zr ratios or synthesis temperatures. The total acid amounts in different UiO-66 samples were consistent with the amount of the missing linkers in their framework.

Table 2. Acid properties of the different UiO-66 samples.

\begin{tabular}{cccc}
\hline Sample & $\begin{array}{c}\text { Peak temperature } \\
\left({ }^{\circ} \mathrm{C}\right)\end{array}$ & $\begin{array}{c}\text { Area under } \mathrm{NH}_{3} \text {-TPD } \\
\text { peaks (a.u./mg) }\end{array}$ & $\begin{array}{c}\text { Total } \mathrm{NH}_{3} \text {-TPD } \\
\text { peak area (a.u./mg) }\end{array}$ \\
\hline UiO-66-100-1 & 118 & $5.51(47.4)$ & 11.62 \\
\hline
\end{tabular}


UiO-66-100-2

Parenthesis is the percentage of contribution of each peak.

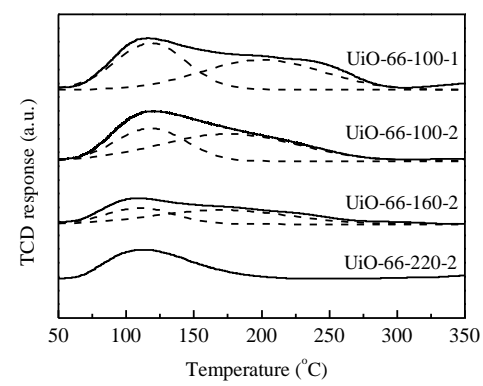

Figure 5. $\mathrm{NH}_{3}$-TPD profiles of the different UiO-66 samples.

\subsection{Catalytic properties}

\subsubsection{Transesterification of tributyrin with methanol over the different UiO-66 samples}

At first, the catalytic performances of UiO-66 prepared at different synthesis conditions were tested using transesterification of tributyrin with methanol, a model reaction for biodiesel synthesis. The transesterification of tributyrin $(\mathrm{T})$ with methanol $(\mathrm{M})$ proceeds in three consecutive steps as shown in the following reaction equations:

$$
\begin{aligned}
& \mathrm{T}+\mathrm{M} \stackrel{k_{1}}{\longrightarrow} \mathrm{D}+\mathrm{MB} \\
& \mathrm{D}+\mathrm{M} \stackrel{k_{2}}{\longrightarrow} \mathrm{Mo}+\mathrm{MB} \\
& \mathrm{Mo}+\mathrm{M} \stackrel{k_{3}}{\longrightarrow} \mathrm{G}+\mathrm{MB}
\end{aligned}
$$

where $\mathrm{D}, \mathrm{MB}, \mathrm{Mo}$, and G denote dibutyrin, methyl butyrate, monobutyrin and glycerol, respectively. As shown in Table 3, the catalytic performances of the different UiO-66 samples in transesterification of tributyrin with methanol are closely related with their acid properties. UiO-66-100-1 with more acid sites and higher acid strength showed the highest activity and its conversion reached $99.3 \%$ after $5 \mathrm{~h}$, while UiO-66-220-2 just gave a conversion of 38.8\% after the same reaction time. Moreover, UiO-66-100-1 
could efficiently transform tributyrin into methyl butyrate and glycerol by three consecutive steps. Combined with the $\mathrm{NH}_{3}$-TPD result, it is difficult to draw a direction relation between the catalytic activity and acid amount/acid strength, but it is obvious that the sample with more acid sites and higher acid strength is favorable for the transesterification reaction, especially for methyl butyrate formation, as evidenced by the increase of the glycerol yield.

The effects of the catalyst amount, reaction time, reaction temperature as well as the methanol to tributyrin (M/T) molar ratio on the catalytic behavior of UiO-66 were investigated over UiO-66-100-1. As shown in Table 3, the tributyrin conversion significantly increased from 6.3\% (blank experiment) to $99.3 \%$ as the catalyst amount increased from 0 to $0.1 \mathrm{~g}$. As the catalyst amount was beyond $0.1 \mathrm{~g}$, the tributyrin conversion did not further increase with the catalyst amount. Different from tributyrin conversion, product distribution always changed with the catalyst amount in the investigated range, and glycerol yield increased with the increase of the catalyst amount.

Table 3. The catalytic results obtained over different UiO-66 samples.

\begin{tabular}{cccccc}
\hline \multirow{2}{*}{ Sample } & Catalyst & Conversion & \multicolumn{3}{c}{ Yeild (\%) } \\
\cline { 4 - 6 } & amount $(\mathrm{g})$ & $(\%)$ & Dibutyrin & Monobutyrin & Glycerol \\
\hline Balnk & 0 & 6.3 & 6.3 & 0 & 0 \\
UiO-66-100-1 & 0.025 & 32.3 & 18.5 & 8.6 & 5.2 \\
UiO-66-100-1 & 0.05 & 54.3 & 29.9 & 12.9 & 11.5 \\
UiO-66-100-1 & 0.1 & 99.3 & 13.5 & 18.4 & 67.4 \\
UiO-66-100-1 & 0.15 & 99.5 & 8.5 & 15.2 & 75.8 \\
UiO-66-100-2 & 0.1 & 81.9 & 38.9 & 10.7 & 32.3 \\
UiO-66-160-2 & 0.1 & 42.2 & 16.3 & 6.2 & 19.8 \\
UiO-66-220-2 & 0.1 & 38.8 & 20.5 & 8.6 & 9.7 \\
\hline
\end{tabular}

Reaction conditions: $120^{\circ} \mathrm{C}, 1.3 \mathrm{mmol}$ tributyrin, $52 \mathrm{mmol}$ methanol, $5 \mathrm{~h}$.

The conversion of tributyrin and the product distribution as a function of reaction time is shown in Figure 6 . When reaction time was prolonged from $1 \mathrm{~h}$ to $2 \mathrm{~h}$, the conversion of tributyrin was increased considerably from $23.9 \%$ to $86.2 \%$, and then with the increase of the reaction time, the increasing degree significantly decreased. The yields of the different products showed different changing trends with the 
reaction proceeding. The glycerol yield always increased in the investigated time range. The dibutyrin and monobutyrin yields increased with reaction time in the initial $2 \mathrm{~h}$, and then the monobutyrin yield basically kept constant, but the dibutyrin yield gradually decreased. Combined with the changing pattern of conversion, it can be inferred that transformation of dibutyrin to glycerol and methyl butyrate is the main reaction occurred in the reaction system after $2 \mathrm{~h}$.

Figure 7 shows the influence of the reaction temperature on the tributyrin conversion and product distribution. The increase of the reaction temperature from $90{ }^{\circ} \mathrm{C}$ to $120{ }^{\circ} \mathrm{C}$ caused a great increase of the catalyst activity. Even if the conversion of tributyin reached nearly $100 \%$ at $120{ }^{\circ} \mathrm{C}$, further increasing reaction temperature still influenced the product distribution. As shown in Figure 7, the yield of glycerol enhanced while the yields of dibutyrin and monobutyrin reduced with the increase of reaction temperature.

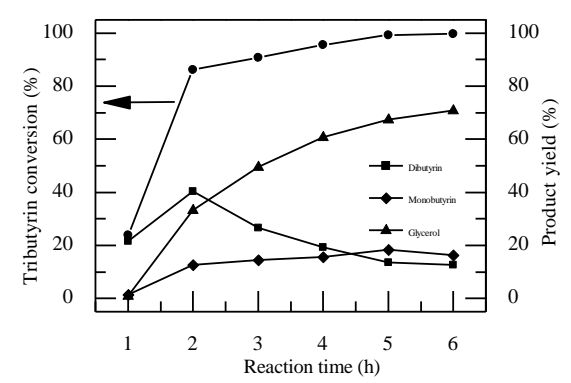

Figure 6. The conversion of tributyrin and the product distribution as a function of reaction time over UiO-66-100-1 (Reaction conditions: $0.1 \mathrm{~g}$ catalyst, $120^{\circ} \mathrm{C}, 1.3 \mathrm{mmol}$ tributyrin, $52 \mathrm{mmol}$ methanol).

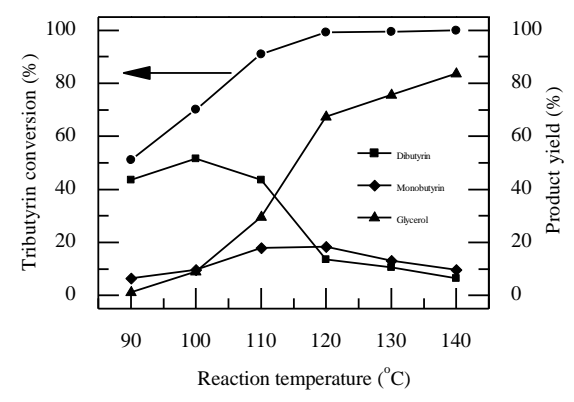

Figure 7. The conversion of tributyrin and the product distribution as a function of reaction temperature over UiO-66-100-1 (Reaction conditions: $0.1 \mathrm{~g}$ catalyst, $0.13 \mathrm{mmol}$ tributyrin, $52 \mathrm{mmol}$ methanol, $5 \mathrm{~h}$ ).

The conversions of tributyrin and product distributions at different M/T ratios are shown in Figure 8. The tributyrin conversion increased with the increase of M/T ratio when the M/T ratio was below 40 . When the ratio was above 40, the increase in tributyrin conversion was not significant. This demonstrates that excess methanol is needed even though theoretically the stoichiometry of the reaction 
requires 3 mol of methanol per mol of tributyrin. This is generally ascribed to the chemical equilibrium shift. Comparatively speaking, the $\mathrm{M} / \mathrm{T}$ molar ratio has more significant influence on the product distribution. When the M/T molar ratio was increased from 10 to 50, the yield of dibutyrin decreased considerably from 50 to $11.5 \%$. This result indicated that dibutyrin was further transferred into glycerol and methyl butyrate at a higher M/T molar ratio.

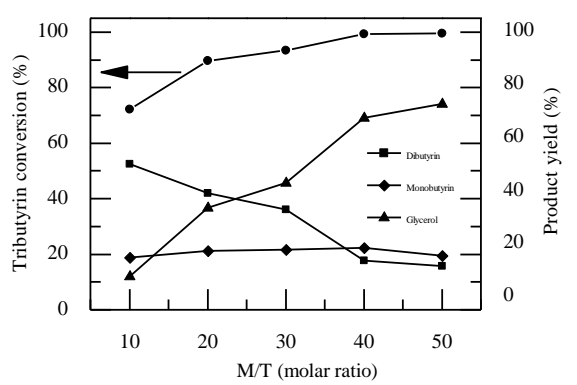

Figure 8. Effect of $M / T$ molar ratios on the transesterification reaction of tributyrin catalyzed by UiO-66-100-1 (Reaction conditions: $0.1 \mathrm{~g}$ catalyst, $120{ }^{\circ} \mathrm{C}, 1.3 \mathrm{mmol}$ tributyrin, $5 \mathrm{~h}$ ).

The reusability of the catalyst was also tested over UiO-66-100-1. For each recycle, the catalyst was recovered by centrifugation and washing with methanol, and then used for the subsequent reactions. From Figure 9, it can be seen that even if the yield of dibutyrin/glycerol increased/decreased with the increase of the recycling number, the reused catalyst still retained $96 \%$ of its original activity after the fourth run. The result indicated that the prepared UiO-66 had a relative high stability under the employed reaction conditions and could be recycled.

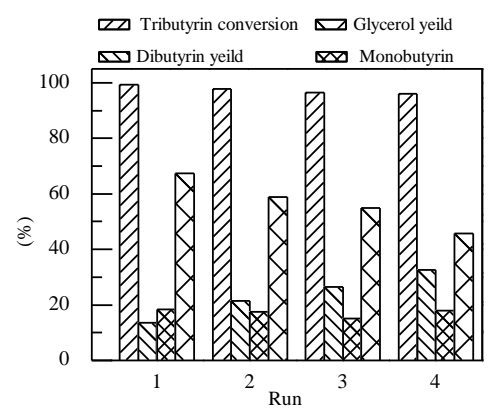

Figure 9. Reuse of UiO-66-100-1 for the transesterification of tributyrin with methanol (Reaction conditions: $0.1 \mathrm{~g}$ catalyst, $120^{\circ} \mathrm{C}, 1.3 \mathrm{mmol}$ tributyrin, $52 \mathrm{mmol}$ methanol, $5 \mathrm{~h}$ ).

\subsubsection{Transesterification of soybean oil with methanol}

To further reveal the catalytic performance of UiO-66 in practical application, the transesterification 
of soybean oil with methanol was investigated over UiO-66-100-1. As shown in Table 4, the UiO-66 catalyst exhibited excellent catalytic performance for the transesterification of soybean oil with methanol. The conversion of soybean oil could reach $98.5 \%$ after reaction $5 \mathrm{~h}$ at $140{ }^{\circ} \mathrm{C}$, and the main product was methyl oleate. This result is comparable to other heterogeneous solid acid catalysts reported in the literatures (Table 4).

Table 4. Transesterification of soybean oil over the different heterogeneous catalysts.

\begin{tabular}{|c|c|c|c|c|c|c|}
\hline Catalysts & $\begin{array}{c}\text { MeOH/oil } \\
\text { (molar ratio) }\end{array}$ & $\begin{array}{l}\text { Oil/cat. } \\
\text { (g/g) }\end{array}$ & $\begin{array}{c}t \\
\text { (h) }\end{array}$ & $\begin{array}{c}T \\
\left({ }^{\mathrm{o}} \mathrm{C}\right)\end{array}$ & $\begin{array}{c}\text { Convers } \\
\text { ion } \\
(\%)\end{array}$ & Ref. \\
\hline $\begin{array}{c}\mathrm{WO}_{3} / \mathrm{ZrO}_{2}, \mathrm{ZrO}_{2} / \mathrm{Al}_{2} \mathrm{O}_{3}, \\
\mathrm{SO}_{4}{ }^{2-} / \mathrm{SnO}_{2}\end{array}$ & 40 & 15 & 20 & $\begin{array}{c}200-3 \\
00\end{array}$ & 90.0 & [31] \\
\hline La/zeolite beta & 14.5 & 61 & 4 & 160 & 48.9 & [32] \\
\hline ETS-10 & 6 & 9 & 24 & 120 & 94.6 & [33] \\
\hline UiO-66 & 40 & 9 & 5 & 140 & 98.5 & $\begin{array}{l}\text { this } \\
\text { work }\end{array}$ \\
\hline
\end{tabular}

\section{Conclusions}

To summarize, in the present work we have demonstrated that zirconium-containing MOF UiO-66 is active, stable and reusable heterogeneous acid catalyst for the transesterification of tributyrin or soybean oil with methanol. The catalytic performances of UiO-66 materials greatly depended on the linker defects. The UiO-66 material had a relative high stability under the employed reaction conditions and could be recycled.

\section{References:}

[1] A. Corma, S. Iborra, A. Velty, Chem.Rev. 107 (2007) 2411-2502.

[2] G.W. Huber, S. Iborra, A. Corma, Chem.Rev. 106 (2006) 4044-4098.

[3] K. Georgogianni, A. Katsoulidis, P. Pomonis, G. Manos, M. Kontominas, Fuel Process.Technol. 90 (2009) 1016-1022.

[4] A. Silitonga, H. Masjuki, T. Mahlia, H. Ong, A. Atabani, W. Chong, Renew.Sust.Energ.Rve 24 
(2013) 514-533.

[5] R. Jothiramalingam, M.K. Wang, Ind. Eng. Chem. Res. 48 (2009) 6162-6172.

[6] Y. Wang, S. Ou, P. Liu, F. Xue, S. Tang, J. Mol. Catal. A: Chem. 252 (2006) 107-112.

[7] M. Canakci, J. Van Gerpen, T.Asae 44 (2001) 1429.

[8] X. Zhang, F. Su, D. Song, S. An, B. Lu, Y. Guo, Appl. Catal. B-environ 163 (2015) 50-62.

[9] E. Lotero, Y. Liu, D.E. Lopez, K. Suwannakarn, D.A. Bruce, J.G. Goodwin, Ind. Eng. Chem. Res. 44 (2005) 5353-5363.

[10] J. Jitputti, B. Kitiyanan, P. Rangsunvigit, K. Bunyakiat, L. Attanatho, P. Jenvanitpanjakul, Chem. Eng. J. 116 (2006) 61-66.

[11] D.E. Lopez, K. Suwannakarn, D.A. Bruce, J.G. Goodwin, J. Catal. 247 (2007) 43-50.

[12] K. Suwannakarn, E. Lotero, K. Ngaosuwan, J.G. Goodwin Jr, Ind. Eng. Chem. Res. 48 (2009) 2810-2818.

[13] E. Andrijanto, E. Dawson, D. Brown, Appl. Catal. B-environ 115 (2012) 261-268.

[14] S. Kitagawa, R. Kitaura, S.i. Noro, Angew. Chem. Int. Ed. 43 (2004) 2334-2375.

[15] A.H. Chughtai, N. Ahmad, H.A. Younus, A. Laypkov, F. Verpoort, Chem. Soc. Rev. 44 (2015) 6804-6849.

[16] G. Férey, Chem. Soc. Rev. 37 (2008) 191-214.

[17] J.H. Cavka, S. Jakobsen, U. Olsbye, N. Guillou, C. Lamberti, S. Bordiga, K.P. Lillerud, J. Amer. Chem. Soc. 130 (2008) 13850-13851.

[18] V. Guillerm, F. Ragon, M. Dan - Hardi, T. Devic, M. Vishnuvarthan, B. Campo, A. Vimont, G. Clet, Q. Yang, G. Maurin, Angew. Chem. Int. Ed. 51 (2012) 9267-9271.

[19] H. Furukawa, F. Gándara, Y.-B. Zhang, J. Jiang, W.L. Queen, M.R. Hudson, O.M. Yaghi, J. Amer. Chem. Soc. 136 (2014) 4369-4381.

[20] J.E. Mondloch, W. Bury, D. Fairen-Jimenez, S. Kwon, E.J. DeMarco, M.H. Weston, A.A. Sarjeant, S.T. Nguyen, P.C. Stair, R.Q. Snurr, J. Amer. Chem. Soc. 135 (2013) 10294-10297.

[21] J. Kim, S.-N. Kim, H.-G. Jang, G. Seo, W.-S. Ahn, Appl. Catal. A-Gen. 453 (2013) 175-180.

[22] F. Vermoortele, R. Ameloot, A. Vimont, C. Serre, D. De Vos, Chem. Commun. 47 (2011) $1521-1523$.

[23] F. Vermoortele, M. Vandichel, B. Van de Voorde, R. Ameloot, M. Waroquier, V. Van Speybroeck, D.E. De Vos, Angew. Chem. Int. Ed. 51 (2012) 4887-4890. 
[24] F. Cirujano, A. Corma, F.L. i Xamena, Catal. Today 257 (2015) 213-220.

[25] F. Cirujano, A. Corma, F.L. i Xamena, Chem. Eng. Sci. 124 (2015) 52-60.

[26] C. Zlotea, D. Phanon, M. Mazaj, D. Heurtaux, V. Guillerm, C. Serre, P. Horcajada, T. Devic, E. Magnier, F. Cuevas, Dalton.Trans. 40 (2011) 4879-4881.

[27] F. Vermoortele, B. Bueken, G.1. Le Bars, B. Van de Voorde, M. Vandichel, K. Houthoofd, A. Vimont, M. Daturi, M. Waroquier, V. Van Speybroeck, J. Amer. Chem. Soc. 135 (2013) 11465-11468.

[28] M. Vandichel, J. Hajek, F. Vermoortele, M. Waroquier, D.E. De Vos, V. Van Speybroeck, Crystengcomm, 17 (2015) 395-406.

[29] G.C. Shearer, S. Chavan, J. Ethiraj, J.G. Vitillo, S. Svelle, U. Olsbye, C. Lamberti, S. Bordiga, K.P. Lillerud, Chem. Mater. 26 (2014) 4068-4071.

[30] J.H. Cavka, S. Jakobsen, U. Olsbye, N. Guillou, C. Lamberti, S. Bordiga, K.P. Lillerud, J. Amer. Chem. Soc. 130 (2008) 13850-13851.

[31] S. Furuta, H. Matsuhashi, K. Arata, Catal. Commun. 5 (2004) 721-723.

[32] Q. Shu, B. Yang, H. Yuan, S. Qing, G. Zhu, Catal. Commun. 8 (2007) 2159-2165.

[33] G.J. Suppes, M.A. Dasari, E.J. Doskocil, P.J. Mankidy, M.J. Goff, Appl. Catal. A-Gen. 257 (2004) 213-223. 


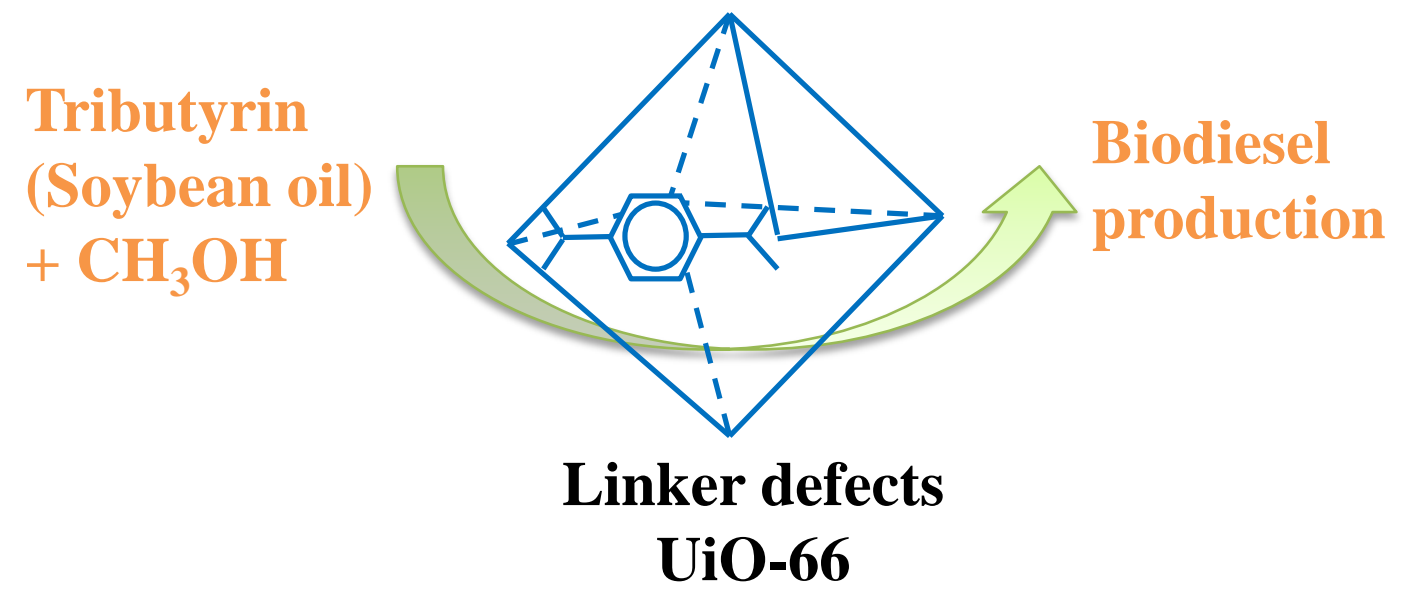

UiO-66 is an efficient and reused catalyst for transesterification of tributyrin and soybean oil with methanol. 